\title{
Walking bleach with sodium perborate tetrahydrate: A safer material and method
}

\author{
Divya S. Sharma ${ }^{1, *}$, Gautami Upadhyay ${ }^{2}$, Bhawna Aidasani ${ }^{3}$, Poorva Goyal ${ }^{4}$ \\ ${ }^{1} \mathrm{HOD},{ }^{2-4}$ Post Graduate Student, Dept. of Pedodontics and Preventive Dentistry, Modern Dental College \& Research Centre, \\ Indore, Madhya Pradesh, India
}

*Corresponding Author:

Email: drdivyassharma@gmail.com

\begin{abstract}
In the era of minimum invasive dentistry and increased demand of esthetics, intra-coronal bleaching in an intact tooth is the most successful yet conservative method. Nonetheless oxidative materials such as hydrogen peroxide, carbamide peroxide, sodium perborate (monohydrate, trihydrate, tetrahydrate) used in combination or alone mixed with water for the same may create complications such as external root resorption. This case report illustrate the use of a bio-friendly material i.e. sodium perborate tetrahydrate with walking bleach technique.
\end{abstract}

Keywords: Dental bleaching, Sodium perborate, Walking bleach.

\section{Introduction}

In today's society, esthetics and good looks have become a major concern, even for a child. Children even at a younger age are aware of the importance of a good smile. Childhood is a period when maximum number of dental traumas occur, while playing at school or at home or due to a fall, etc. For a pedodontist, cases of trauma and pulpal infections are routine procedures. ${ }^{1}$ Such traumas may lead to pulp necrosis and followed by tooth discoloration for which a patient visit to the dentist.

Intra-coronal bleaching with Walking Bleach technique, is a widely accepted procedure when it comes to the treatment of such an anterior discolored teeth. ${ }^{2}$ It was introduced by Spasser ${ }^{3}$ and later modified by Nutting and Poe. ${ }^{4}$ It is done on an endodontically treated tooth and is a non-invasive conservative procedure compared to crowns and veneers as they require reduction of the clinical crown of the tooth. ${ }^{5}$ Recent intra-coronal bleaching materials are oxidative in nature and depend on concentration and amount of released hydrogen peroxide from parent material i.e. Hydrogen peroxide gel/ liquids, carbamide peroxide or sodium perborate. ${ }^{6}$ These agents can either be used in combination or alone mixed with water. ${ }^{7}$

When hydrogen peroxide $\left(\mathrm{H}_{2} \mathrm{O}_{2}\right)$ based material is used as an intra-coronal beaching agent, the haem present in the root canal, as a result of intra-pulpal bleeding at the time of trauma, gets converted into ferrous form. This ferrous reacts with hydrogen peroxide to undergo Fenton reaction. ${ }^{10}$ Fenton reaction is an exothermic catalytic reaction that converts $\mathrm{H}_{2} \mathrm{O}_{2}$ into highly reactive hydroxyl/ per-hydroxylide radicals/ ions. ${ }^{10,-12}$ These reaction products are called Reactive Oxygen Species (ROS). The use of concentrated $\mathrm{H}_{2} \mathrm{O}_{2}$ combined with thermal technique as it is in in-office bleach, generate ample amount of ROS. This ROS may penetrate through dentinal tubule and then into extraradicular space causing external root resorption. ${ }^{10}$
Studies ${ }^{13,14}$ have shown the presence of $\mathrm{H}_{2} \mathrm{O}_{2}$ molecules and hydroxyl radicals around the root surface while intra-coronal bleaching with oxidative materials. Therefore choice of material and the technique should be able to control the release of ROS as well as be able to reduce the penetration of these into periradicular space.

Sodium perborate (SP) has not shown any such complication ever since its introduction. ${ }^{10,15}$ This article presents a successful intra-coronal bleaching procedure done by SP with one year follow up.

\section{Case Report}

A 10 year old male patient reported to the Department of Pedodontics and Preventive Dentistry, Modern Dental College \& Research Centre, Indore, India, with a chief complaint of blackening of tooth in the upper front tooth region (Fig. 1). He gave a history of trauma during playing at school one year ago. There was no history of intraoral or extra oral bleeding at the time of trauma. The patient did not have any discomfort as the pain or the sensitivity. Oral examination revealed discolored 21 with no fracture involved and the tooth was intact. There was no sinus, swelling or pain on percussion on examination with respect to 21 but greyish-black discoloration was apparent. Radiographically, there was no periapical lesion or any adverse change with respect to 21 . The patient did not respond to heat test and customised pulse-oximeter ${ }^{16}$ gave a reading below 80 , both suggestive of pulpal necrosis. Hence root canal treatment (RCT) followed by intra-coronal bleaching with 21 was planned. A method given by Sharma DS et al. was followed. ${ }^{10}$ 


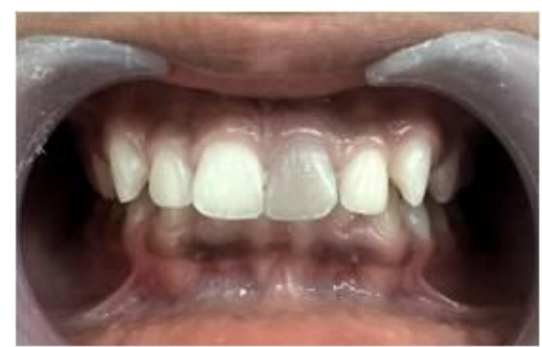

Fig. 1: Discolored 21 due to previous trauma

During first appointment, the shade of the tooth was matched with the adjacent teeth using Vita Shade Guide. After the RCT was completed, the procedure to apply GIC (glass ionomer cement) as a barrier was performed. For this the clinical length of crown was measured using a calibrated periodontal probe from incisal edge to the base of sulcus and then $4 \mathrm{~mm}$ were added to this measured length. After this gutta percha was removed till this length with the help of hot pluggers and peizo-reamers. Thereafter GIC was placed over the gutta percha of about $4 \mathrm{~mm}$ thickness corresponding to the gingival attachment. The base was then shaped by reducing it with a bur in such a way that it had its dome shape facing the labial wall and proximally upto the sulcus base and incisal to the CEJ; but beyond the sulcus base lingually. ${ }^{10}$ This was checked by radiographs for confirming the desired shape of the base (Fig. 2).

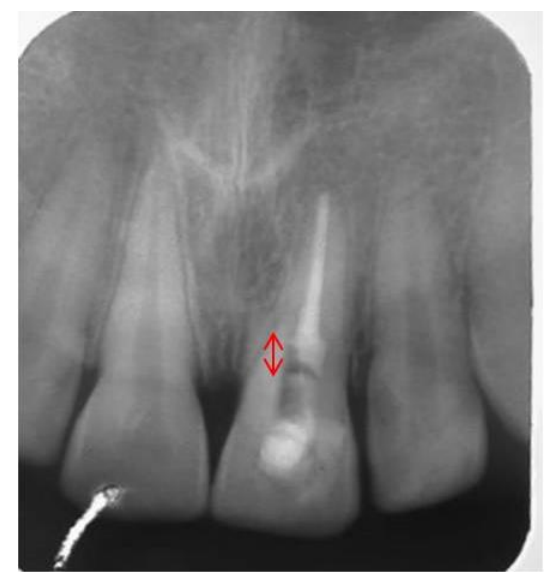

Fig. 2: IOPA of 21 shows width and length of GIC barrier on gutta-percha

Once base was properly applied, the process for bleaching agent application was done. SP tetrahydrate (Loba chemie Pvt. Ltd., India) was mixed with distilled water in a thick paste like consistency as it is easier to carry to the cavity without any spillage. This paste was then applied to the labial surface of the access cavity. Temporary filling was done with IRM (Caulk, DENTSPLY).

Patient was recalled on the $7^{\text {th }}$ day and slight lightning in the dark shade of the tooth was noticed. Same procedure was repeated and the patient was recalled after a week. On the second recall only, the shade of 21 matched with the adjacent teeth showing a better efficiency of SP mixed with distilled water. Whole procedure was repeated for one more week to make 21 one shade lighter so that if the shade gets darker as compared to its adjacent teeth with time, the shade of the tooth returns to normal and preventing it from further darkening. After obtaining the desired shade of 21, a cotton pellet wetted in distilled water was put over in access cavity to remove the remaining ROS (as they hinder the polymerization reaction of composite that is used as a permanent filling) and maintain the hydration of the dentine. ${ }^{10}$ After this the access caity was sealed with final composite restoration (Fig. 3).

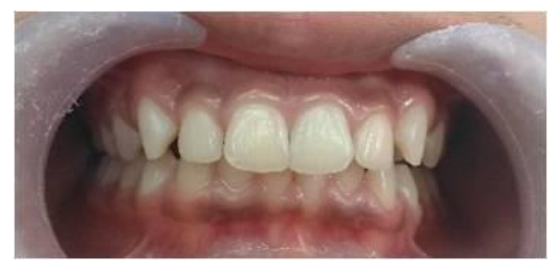

Fig. 3: Successful bleaching and happy smile after 3 weeks of walking bleach

Patient had showed no recurrence of discoloration and other problems at one year of follow up. This proved that SP is not only efficient but also a safer and cost effective bleaching agent.

\section{Discussion}

The chances of a bleaching agent penetrating the peri-radicular region and causing root resorption mainly depend on the anatomical features of the tooth. ${ }^{10}$ However it is preferred that bleaching should be confined only to the supragingival chamber and that the dentinal tubules need to be protected by the use of base that acts as a barrier. Sherwood AI et al. ${ }^{17}$ stated that the at least a 3-4 $\mathrm{mm}$ thick base should be applied to prevent the bleaching chemicals from percolating in the periodontal region.

Studies have shown that walking bleach done with SP did not show external resorption even after 2-7years of the procedure. ${ }^{18}$ While histological findings showed root resorption and ankylosis caused by $\mathrm{H}_{2} \mathrm{O}_{2}$ as a bleaching agent. ${ }^{19}$ And in case the dentin thickness is less in between the periodontium and the prepared canal spaces, $\mathrm{H}_{2} \mathrm{O}_{2}$ showed increased root resorption.

The vital tissues need to be protected, and for this reason tetrahydrate from of SP was used (not monohydrate or trihydrate forms) as it is alkaline and remain active for 6 days which is compatible with appointment schedules. ${ }^{10}$ Its alkaline $\mathrm{pH}$ is due to its controlled release of hydroxyl radicals. ${ }^{20}$ The rate of fenton reaction and hence the formation of ROS also get controlled as these are dependent on the release of hydrogen peroxide from parent material (SP). This rate is reduced in alkaline medium as in SP tetrahydrate. ${ }^{21,22}$ 
Sharma and Sharma ${ }^{22}$ found that there is release of more amount of per-hydroxyl radical compared to the hydroxyl radicals from sodium perborate tetrahydrate, which again confirms its bio-friendly nature.

\section{Conclusion}

This article presented a successful intra-coronal bleaching procedure carried out on a young permanent tooth with a milder bleaching agent i.e. SP tetrahydrate mixed with distilled water. This makes this technique easier, efficient, safer and cost effective. Hence from the biologic point of view, the use of SP tetrahydrate with distilled water is recommended because of its biocompatibility and user friendly features for both the child and the dentist.

Funding: No funding sources.

Conflict of interest: None declared.

\section{References}

1. Bussadori SK, Faynna Roth F, Cardoso Guedes C, Fernandes KP, Domingues MM, Wanderley MT. Bleaching non vital primary teeth: case report. J Clin Pediatr Dent 2006;30(3):179-82.

2. Chng HK, Palamara JEA, Messer HH. Effect of hydrogen peroxide and sodium perborate on biomechanical properties of human dentin. J Endodontic 2002;28(2):627.

3. Spasser HF. A simple bleaching technique using sodium perborate. N Y State Dent J 1961;27:332-34.

4. Nutting EB, Poe GS. A new combination for bleaching teeth. J South Calif Dent Assoc 1963;31:289-91.

5. Attin T, Paque F, Ajam F, Lennon AM. Review of the current status of tooth whitening with the walking bleach technique. Int Endodontic J 2003;36:313-29.

6. Dahl JE, Pallesen U. Tooth bleaching - a critical review of the biological aspects. Crit Rev Oral Biol Med 2003;14(4):292-304.

7. Rotstein I, Zalkind M, Mor C, Tarabeh A, Friedman S. In vitro efficacy of sodium perborate preparations used for intra-coronal bleaching of discolored non-vital teeth. Endod Dent Traumatol 1991;7:177-80.

8. Latcham NL. Postbleaching cervical resorption. J Endod 1986;12:262-64.

9. Friedman S, Rotstein I, Libfeld H, Stabholz A, Heling I. Incidence of external root resorption and esthetic results in 58 bleached pulpless teeth. Endod Dent Traumatol 1988;4:23-6.
10. Sharma DS, Barjatya K, Agrawal A. Intra-coronal bleaching in young permanent and primary tooth with biologic perspectives. $J$ Clin Pediatr Dent 2011;35(4):357-60.

11. Zepp RG, Faust BC, Holgne J. Hydroxyl radical formation in aqueous reactions ( $\mathrm{pH}$ 3-8) of iron with hydrogen peroxide: The photo-fenton reaction. Environ Sci Technol. 1992;26(2):313-19.

12. Fenton reactions. J Microsc Ultrastruct 2017.

13. Rotstein I, Torek Y, Misgav R. Effect of cementum defects on radicular penetrations of $30 \% \mathrm{H}_{2} \mathrm{O}_{2}$ during intracoronal bleaching. J Endod 1991;17(5):230-33.

14. Sharma DS, Sharma S, Natu SM, Chandra S. An in vitro evaluation of radicular penetration of hydrogen peroxide from bleaching agents during intra-coronal tooth bleaching with an insight of biologic response. J Clin Pediatr Dent 2011;35(3):289-94.

15. Spasser HF. A simple bleaching technique using SP. NY State Dent J 1961;27:332-34.

16. Sharma DS, Misra S, Banda NR, Vaswani S. In vivo evaluation of customized pulse oximeter and sensitivity pulp tests for assessment of pulp vitality. J Clin Pediatr Dent 2018;43(1).

17. Sherwood AI, Miglani RS, Lakshminarayan L. Efficacy of glass ionomer as a barrier material in non-vital bleaching -A stereomicroscopic study. Endod 2004;16:12-5.

18. Harrington GW, Natkin E. External resorption associated with bleaching of pulpless teeth. $J$ Endod 1979;5:344-48.

19. Madison S, Walton R. Cervical root resorption following bleaching of endodontically treated teeth. $J$ Endod 1990;16:570-74.

20. Ari H, Ungor M. In vitro comparision of different types of SP used for intracoronal bleaching of discoloured teeth. Int Endod J 2002;23:167-69.

21. Weiger R, Kuhn A, Lost C. Effect of various types of SP on the ph of bleaching agents. J Endod 1993;19(5):239-41.

22. Sharma H, Sharma DS. Detection of hydroxyl and perhydroxyl radical generation from bleaching agents with Nuclear Magnetic Resonance spectroscope. J Clin Pediatr Dent 2017;41(2):126-35.

How to cite the article: Sharma D., Upadhyay G., Aidasani B., Goyal P. Walking bleach with sodium perborate tetrahydrate: A safer material and method. Ann Prosthodont Restor Dent 2018;4(3):76-78. 